September 8, $2009 \quad$ 16:2 main

International Journal of Information Acquisition

(c) World Scientific Publishing Company

\title{
INFORMATION ACQUISITION USING EYE-GAZE TRACKING FOR PERSON-FOLLOWING WITH MOBILE ROBOTS
}

\author{
HEMIN OMER LATIF ${ }^{*}$ \\ Department of Computing and Technology, Nottingham Trent University, \\ Nottingham, NG11 8NS, United Kingdom, \\ E-mail: hemin.latif@ntu.ac.uk \\ NASSER SHERKAT \\ Department of Computing and Technology, Nottingham Trent University, \\ Nottingham, NG11 8NS, United Kingdom, \\ E-mail: nasser.sherkat@ntu.ac.uk \\ AHMAD LOTFI \\ Department of Computing and Technology, Nottingham Trent University, \\ Nottingham, NG11 8NS, United Kingdom, \\ E-mail:ahmad.lotfi@ntu.ac.uk
}

Received (to be inserted by publisher)

\begin{abstract}
In the effort of developing natural means for human-robot interaction (HRI), significant amount of research has been focusing on Person-Following (PF) for mobile robots. PF, which generally consists of detecting, recognizing and following people, is believed to be one of the required functionalities for most future robots that share their environments with their human companions. Research in this field is mostly directed towards fully automating this functionality, which makes the challenge even more tedious. Focusing on this challenge leads research to divert from other challenges that coexist in any PF system. A natural PF functionality consists of a number of tasks that are required to be implemented in the system. However, in more realistic life scenarios, not all the tasks required for $\mathrm{PF}$ need to be automated. Instead, some of these tasks can be operated by human operators and therefore require natural means of interaction and information acquisition. In order to highlight all the tasks that are believed to exist in any PF system, this paper introduces a novel taxonomy for PF. Also, in order to provide a natural means for HRI, TeleGaze is used for information acquisition in the implementation of the taxonomy. TeleGaze was previously developed by the authors as a means of natural HRI for teleoperation through eye-gaze tracking. Using TeleGaze in the aid of developing PF systems is believed to show the feasibility of achieving a realistic information acquisition in a natural way.
\end{abstract}

Keywords: Eye-Gaze Tracking, Human-Robot Interaction, Robotic Person-Following, TeleGaze

${ }^{*}$ Present Address 


\section{H. O. Latif, et al.}

\section{Introduction}

In order to enable future robots to interact with their human companions in a variety of different environments with varying working and interacting conditions, Person-Following $(\mathrm{PF})$ is believed to be one of the main required functionalities [Takemura et al, 2007]. Therefore, PF is becoming an increasingly popular research topic in the field of robotics and significant progress towards robust and reliable implementation of this functionality can be observed in the literature [Hu et al, 2007]

Tracking the Person-Of-Interest (POI) and establishing a physical relation between the target and the follower are believed to be the main challenges in any PF application. Tracking the POI is mostly achieved using object tracking algorithms, with or without, some modifications [Chen and Birchfield, 2007]. Keeping the POI within a desired distance from the robot is achieved using different controlling algorithms, where certain functionalities such as obstacle avoidance can also be added [Tsalatsanis et al, 2007]. The focus of most research on $\mathrm{PF}$ has been the challenge of automating these two tasks.

Looking at realistic scenarios and different contexts of applications, there exist a number of other tasks in any PF system which raises a number of other challenges. A complete PF system cannot be achieved in a natural way without implementing all the tasks that coexist with each other. This, however, does not mean that all the tasks in PF need to be automated in order to achieve natural Human-Robot Interaction (HRI). Depending on the context of the application, some of the tasks look more natural when they are not automated, but rather are operated by a human operator. Therefore, automating or operating each task in the PF system is highly application dependent.

Eye contacts and eye communications is one of the natural modes of interaction between human beings [Rutter, 1984]. Continuous advancements in eye tracking technology has resulted in using inputs from human eyes in developing many Human-Computer Interaction (HCI) applications [Duchowski, 2002]. Therefore, HRI applications are not exempted from this technology either [Decher and Piepmeier, 2008]. Due to the belief that eye tracking data is natural representation of human intentions and reactions [Mohammad and Nishida, 2008], they are widely used in developing natural HRI applications with the aid of Intelligent User Interfaces (IUI) [Bhuiyan and Liu, 2007].

In order to address most of the tasks required to be implemented in any PF system, this paper continues previous works by the authors [Latif et al, 2009] in presenting a novel taxonomy for $\mathrm{PF}$. The list of tasks and likely cycles of their implementation are presented in the taxonomy. In order to achieve a rather complete PF system in the form of natural HRI, inputs from human eyes are used to interact with a robotic agent. TeleGaze, which stands for teleoperation through eye gaze, is integrated to an automated PF system implementing most of the tasks presented in the taxonomy in a natural form of HRI. Information acquisition through inputs from the operator's eyes is believed to aid in the naturalness of the established HRI.

To address the issues mentioned above, this paper is organized as follows: before introducing the PF taxonomy, section 2 defines some necessary vocabularies. Then the taxonomy is presented in section 3 with examples of likely scenarios in section 4 . Section 5 covers the implementation of the taxonomy and how the tasks can be implemented using different forms of information acquisition. A brief background on TeleGaze is included in section 6 . In section 7 , the integration of TeleGaze into PF is presented. Section 8 covers algorithms and apparatus used in developing the PF system and conclusions are in section 9 .

\section{Terminology Definitions}

Before digging into the PF taxonomy and the different tasks that are involved in developing any PF system, it is necessary to clarify and define some terminologies that will be used throughout this paper. This is necessary due to the fact that the terms tracking and following are used in the literature to refer to the same meaning and/or different meanings interchange- 
ably [Takemura et al, 2007; $\mathrm{Hu}$ et al, 2007; Hyukseong et al, 2005]. Therefore, in order to standardize the use and the meaning of these two terms in PF applications and future writings, it is necessary to define them in this context.

Tracking is going to be used in the taxonomy to refer to the set of actions taking place in order to keep the POI in the vicinity of the robot without altering the physical position of the robotic platform. This might include digital, optical and physical actions of only the active vision system of the robot and not the whole robotic platform. Digital and/or optical zooming, for example, might be used to keep the appearance of the POI in the scene at a certain ratio of the whole scene. Also pan/tilt might be used to keep the POI in a certain area of the scene.

Following, on the other hand, is going to be used in the taxonomy to refer to the set of actions taking place in order to keep the POI in the vicinity of the robot by altering the physical position of the robotic platform. This, in its basic form, consists of the four common actions of forward, backward, left, and right. This task requires distance information to keep the robot at a desired distance of the moving target while avoiding accidents that might occur if getting to close to the target.

\section{Taxonomy of Person-Following}

The challenge of keeping track of the Person-OfInterest (POI) is believed to be the main challenge in any $\mathrm{PF}$ application. This challenge is mostly addressed through modifying or developing object tracking algorithms used to keep track of the POI [Tsalatsanis et al, 2007]. Or, in some cases, to cope with variations in the interactions' conditions, fusion of cues and algorithms is used to address the problem [Bernardin et al, 2007]. However, a complete PF system is not limited to this challenge only. Regardless of the complexity of the applications and the likely scenarios, a complete PF system consists of a number of tasks that each might raise a number of challenges during the course of interaction and the implementation of the PF functionality.
The aim of the PF taxonomy introduced here is to highlight the tasks involved in developing any PF system. All the tasks presented in the taxonomy are required to be implemented in a natural form of HRI regardless of the application context. In addition to the tasks themselves, the taxonomy presents a number of likely interaction scenarios in the form of Loops-OfInteraction (LOI) where each loop consists of a number of tasks. The complete PF taxonomy is illustrated in Fig. 1. Notice the difference between Person-Following (PF) as the entire system and person-following (pf) as an individual task in the overall system.

The ideal LOI is presented in the taxonomy with thick-continuous lines starting from task one and ending with task eight. However, different loops in the taxonomy represent different interaction scenarios that are likely to happen in any PF application. Although, for instance, it is most likely that task two will start once task one is accomplished, task eight might start instead after task one if a wrong person is registered. Therefore, the LOI that consists only of tasks one and eight is a likely interaction scenario in real life $\mathrm{PF}$ applications. The mentioned scenario explains the importance of the taxonomy and how a PF application needs to address more than just the problem of tracking and following the POI.

\section{Interaction Scenarios}

Scenarios are believed to be very essential in designing any interactive system as they present stories about interactions [Benyon, 2005]. Therefore, in order to provide better understanding of likely interaction scenarios in PF applications, following is two examples of scenarios that, in addition to the tasks of tracking and following the POI, involve other tasks .

As the first example of likely interaction scenarios, a person gets registered in the system as the POI (task one) and the system starts tracking that person (tasks two and three). For some reasons such as change in interest, realizing that a wrong person is registered, or losing the POI the system stops tracking that person (task seven) and the person gets deregistered from the 
September 8, $2009 \quad$ 16:2 main

4 H. O. Latif, et al.

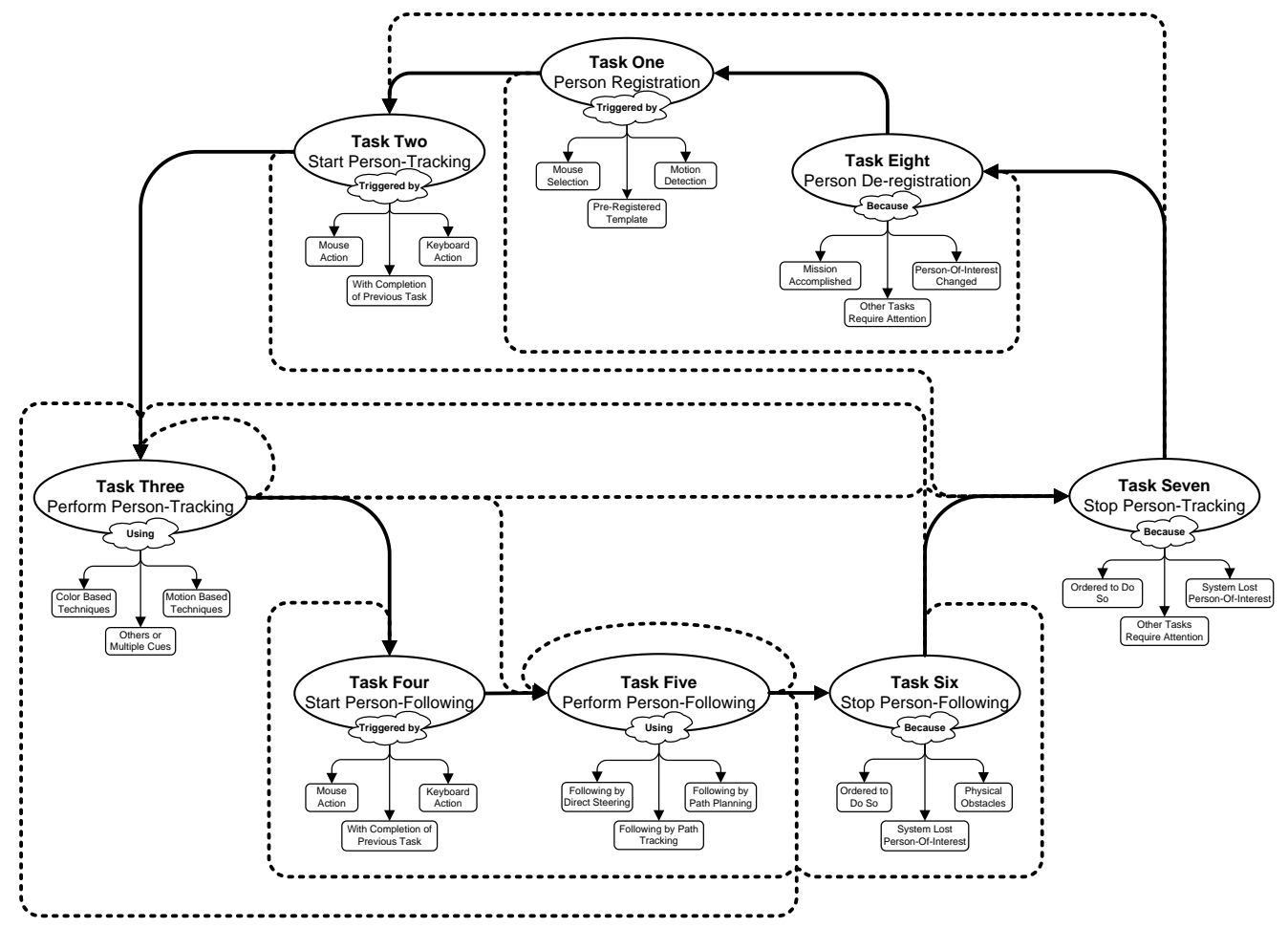

Fig. 1. The taxonomy of PF. Continuous lines present the most likely LOI and dashed lines present possible LOI.

system as the POI (task eight). To continue, the LOI returns back to the task of registering a person (task one) and then any other likely LOI based on the conditions of interaction. The tasks involved in this LOI are illustrated in Fig. 2.

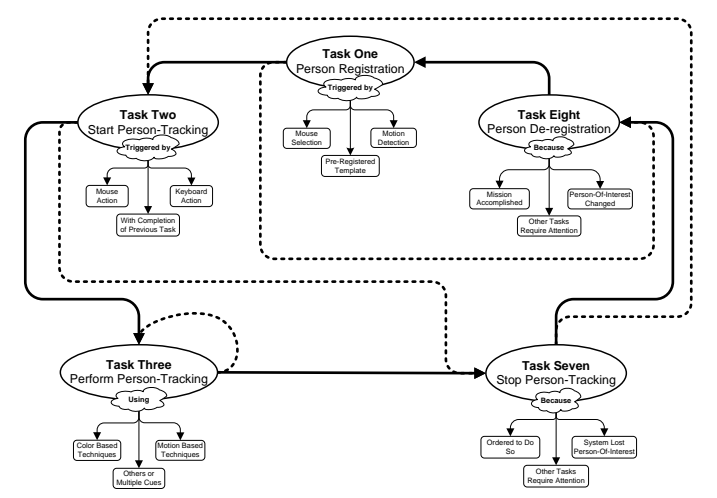

Fig. 2. Tasks and LOI of the first scenario example.

The LOI shows that not all the tasks in the $\mathrm{PF}$ taxonomy were involved in the scenario. In- stead, a realistic interaction scenario, such as this one, could take place without invoking any of the tasks that are related to following the POI (tasks four, five, and six). Furthermore, even within the loop of this interaction scenario there are other possible scenarios that might take place as partial LOI. In summary, only the following tasks were invoked in this interaction scenario:

- Task One (Person Registration)

- Task Two (Start Person-Tracking)

- Task Three (Perform Person-Tracking)

- Task Seven (Stop Person-Tracking)

- Task Eight (Person De-registration)

Another example of an interaction scenario is that a person gets registered in the system as the POI (task one) and the system starts and performs tracking (tasks two and three) and following (tasks four and five) the POI. Then the system stops following the POI (task six) but it still keeps tracking the person (task three). Or, 
it stops tracking the person (task seven) but it keeps the registered person as the POI. In the former case, when the system stops following but keeps tracking the POI, the system waits for restarting the person-following (task four). In the latter case however, the system needs to restart tracking the POI (task two). In both cases, person registration (task one) is not required as the same person is still registered in the system as the POI. The tasks and the LOI of this scenario are illustrated in Fig. 3.

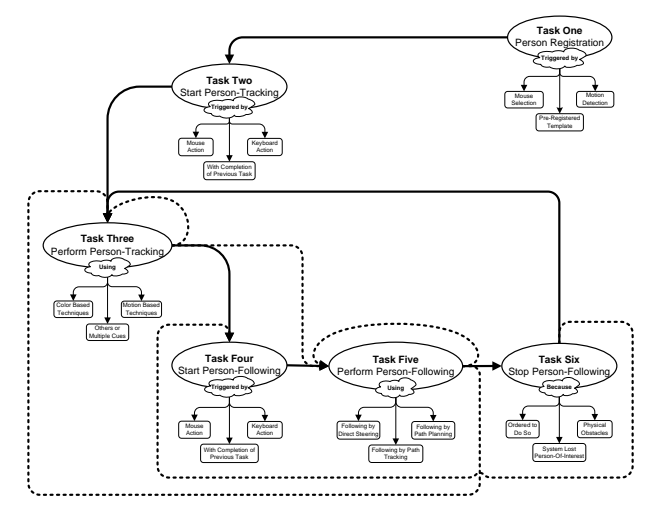

Fig. 3. Tasks and LOI of the second scenario example.

For this example, only the following tasks were invoked:

- Task One (Person Registration)

- Task Two (Start Person-Tracking)

- Task Three (Perform Person-Tracking)

- Task Four (Start Person-Following)

- Task Five (Perform Person-Following)

- Task Six (Stop Person-Following)

The two interaction examples show how a number of tasks in a number of different likely LOI might be involved in a PF scenario. The presentation of the scenarios shows that how care must be taken not to limit the problem span of $\mathrm{PF}$ to the tasks of tracking and following only. Each of the tasks presented in the taxonomy require attention as much as the tasks of tracking and following.

\section{Taxonomy Implementation}

The forms of information acquisition for both the system and the human operator vary de- pending on task requirements. The combination of autonomous and non-autonomous functionalities in one application is a common approach in developing many robotic systems [Carelli et $a l, 2008]$. Some of the tasks in the taxonomy can be either operated, which requires information acquisition for the human operator, or automated. This means that not all the tasks presented in the taxonomy require automation. In fact, some of them make more sense when they are operated by a human operator and not automated. One of the tasks for example that is most likely to require operation and not automation is registering the POI (task one). However, this does not mean that operating the task should be achieved in an artificial way and not considered from a natural HRI point of view. Implementing this task has been achieved in a number of different ways, as reported in the literature, so far such as using a mouse selection, people detection [Treptow et al, 2005], motion detection [Hyukseong et al, 2005], or even a pre-registered template such as a predetermined color of the POI [Tsalatsanis et al, 2007]. This task however, when operated, needs to be implemented in a more natural way of HRI interaction [Spexard et al, 2006].

Also some of the other tasks such as starting person-tracking (task two), starting personfollowing (task four), stopping person-following (task six), stopping person-tracking (task seven) and finally person deregistration (task eight) can be operated in a PF application and not automated. Although some of these tasks are merged into one task in some applications such as starting person-tracking (task two) once the person registered (task one) and then starting personfollowing (task four) once person-tracking (task two) started. However, in a more realistic application each one of these tasks needs to be implemented once the conditions for their implementation are met and not as a group of tasks altogether. Therefore, an ideal PF application needs to deal with invoking each task separately from the other tasks in the taxonomy while it enables a natural HRI form of invoking each task. TeleGaze, which is introduced in the next section, is used as a natural means of HRI in developing and designing a rather realistic PF application. 


\section{H. O. Latif, et al.}

\section{Natural HRI using TeleGaze}

Previously, the authors developed TeleGaze as a means of teleoperation through eye gaze for natural and intuitive means of HRI. TeleGaze uses inputs from human eyes to enable a human operator to navigate a mobile robot from a remote location using an intelligent user interface. The TeleGaze interface enables both monitoring as well as controlling. Monitoring is achieved using real time images from a video camera mounted on the mobile robot. Controlling is achieved using inputs from the human operator's eye to issue motion commands. Both monitoring and controlling are achieved with out any involvements of the operator's hands as TeleGaze is essentially developed to reduce the amount of body engagement in teleoperation applications for mobile robots. If a human operator is able to navigate a mobile robot only using inputs from his/her eyes, then the hands of the operator are free from the navigation task, either partially or fully.

TeleGaze provides information acquisition using a powerful presentation of two layers of information on top of each other. The background layer is the real time images that come back from a video camera mounted onboard of the robot. This layer works as the feedback layer of the robotic platform and the status of the system. The background layer is augmented with a transparent layer in the foreground that enables controlling the robotic platform.

The action regions are transparent regions each associated with a certain action command. Through the action regions, the operator is enabled to issue action commands required to move the robot, control the pan/tilt unit of the camera and control the TeleGaze interface itself. In order to issue a command, the operator needs to look at the action region associated with that particular command for a dwell time period of a third of a second. This is the time it approximately takes two consequent fixations to happen in the same action region. The controlling layer is composed of a number of regions which are called action regions. An illustration of the layout of the TeleGaze interface with captions for each action region is shown in Fig. 4.

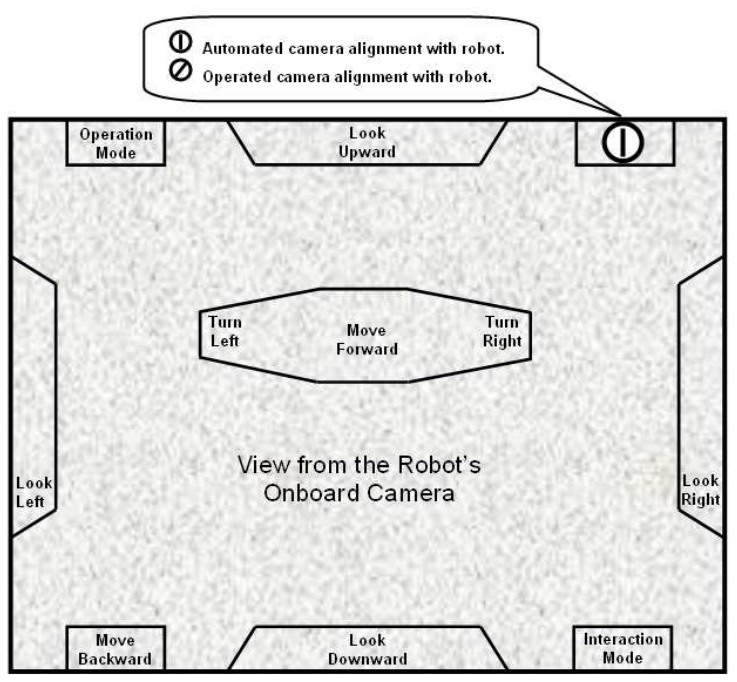

Fig. 4. Layout Illustration of the TeleGaze Interface.

To grab a better image of the two layers of the interface, an actual snapshot of the interface while in work is shown in Fig. 5.

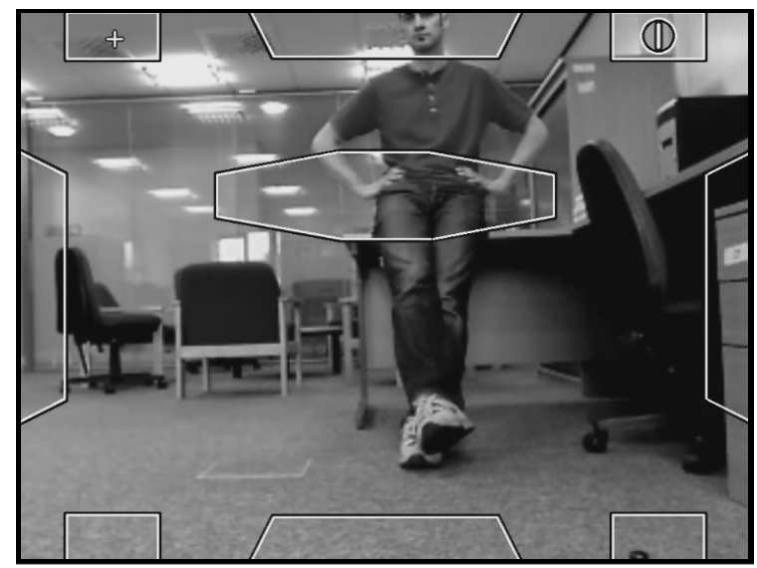

Fig. 5. A Sanpshot of the TeleGaze Interface.

Changing between different modes of interaction and different modes of operation is included in controlling the interface. The two different modes of interaction are the interaction mode and the inspection mode. The interaction mode enables the operator to interact with the robot by issuing motion commands through the use of the action regions. The inspection mode enables the operator to use the interface to inspect the scene without issuing any commands except commands required to switch back to the 
interaction mode. The two different modes of operation are the TeleGaze mode and the PF mode. The TeleGaze mode enables the operator to interact with the robot using inputs from the eyes. The PF mode enables the operator to operate the robot in a PF mode. Once switched to the $\mathrm{PF}$ Mode the operator is enabled to switch back to the TeleGaze Mode using inputs from his/her eyes. A snapshot of the TeleGaze interface in the PF Mode is shown in Fig. 6, where only one action region is available to interact with to switch back to the TeleGaze Mode if desired.

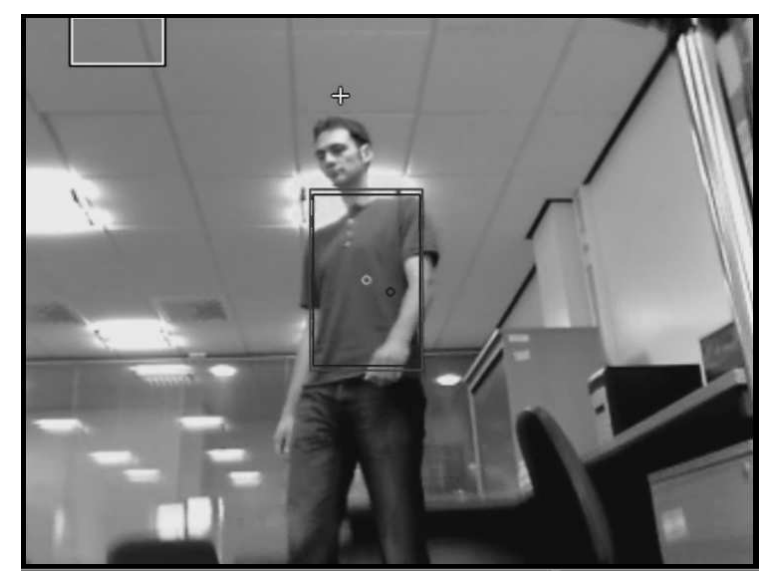

Fig. 6. The TeleGaze Interface in the PF Mode.

For more information on TeleGaze and the TeleGaze interface, the reader is recommended to refer to the authors' previous publications on TeleGaze [Latif et al, 2008a; Latif et al, 2008b].

\section{TeleGaze Integration into PF}

The TeleGaze mode, which is one of the two operation modes of TeleGaze, enables teleoperation through human eye gaze. In other words, the robotic agent reads the intentions of its human partner by tracking its partner's eye movements and corresponds to these eye movements in the form of action commands. The PF mode, however, enables the operator to change from a teleoperated mode to an automated PF mode. This mode, based on the principle of understanding the operator's intentions through eye movement data, enables the operator to select the POI by gazing at him/her for a certain period of time. Gazing at a person in the scene of the robot implicitly indicates that the operator is interested in following that person. This is a natural and intuitive implementation of registering the POI (task one) in the PF system. Once the POI is registered in the system, the system informs the operator by drawing a box surrounding the POI in the scene. When this task is completed, then the system starts tracking and following this person (tasks two, three, four, and five). The dependent functionality of the system based on the interaction and operation modes via the TeleGaze interface is believed to achieve one of the basic principles of natural HRI which is implicit changes in modes of interaction [Goodrich and Olsen, 2003].

The only action region available in the $\mathrm{PF}$ mode is for the operator to gain back control over the robot. To do this, all that required is gazing at the action region which changes the operation mode back to the TeleGaze mode where the operator can control the robot. In other words, stop following and tracking the POI (tasks six and seven) and deregistering the POI (task eight). However, during the course of PF, if the robot lost the POI for any reasons, it keeps looking for him/her for a period of time. If the POI was found, then it starts following him/her again (tasks two, three, four and five). If the robot failed to find the POI, then it switches back to the TeleGaze mode where the operator teleoperates the robot and the POI gets deregistered (task eight). During the course of PF if the POI is lost, the robot keeps the registration of the lost person as the POI unless the operator intervene and change back to the TeleGaze mode or select a different person to be the POI.

\section{Algorithms and Platforms}

A basic version of the Camshift tracking algorithm was modified and implemented in OpenCV [Bradski, 2008] for the task of tracking the POI. The Camshift algorithm enables a color based object tracking in real-time once a color blob is selected from the scene. It is not one of the objectives of this research to develop an object tracking algorithm. However, for the purpose of the $\mathrm{PF}$ application some modifications were made to the Camshift algorithm. Consid- 


\section{H. O. Latif, et al.}

ering the sensitivity of the Camshift algorithm to rapidly changing scenery such as fast movements of the POI from one side of the scene to another, the modifications included expanding the searching span for the POI when (s)he is lost. This expands the functionality of the algorithm to search the whole scene for the POI and hence more chances to find the person if (s)he still exists in any parts of the scene. However, the color blob that represents the POI needs to meet a minimum threshold of $10 \%$ of the image's dimensions in order to be considered found and available for tracking.

A rather interesting modification to the algorithm is calculating the distance from the object been tracked. Solely depending on the images from one single video camera, the distance between the POI and the robot is kept at the initialization threshold. Once the POI is selected from the scene, the algorithm calculates the initial size of the color blob that represents the person. Then it keeps the distance from the person that keeps the color blob at the same initial size in the scene. This means any decrease in the size of the color blob leads to moving the robot towards the person and vice versa. The distance kept between the robot and the POI is highly flexible and depending on the initial distance when the POI is registered to the robot. Therefore, the task of following the person (task five) is implemented with highly natural but simple implementations of vision algorithms.

The experimental platform used in developing TeleGaze consists of a Wi-Fi enabled mobile robot and an active robotic vision subsystem at one end of the system, an eye tracking sub-system at the other end of the system, and the TeleGaze interface running on a $\mathrm{PC}$ located in the remote teleoperation station in the middle of the system. The TeleGaze interface and the software behind it work as a meeting point for the data flow from both ends of the system. TeleGaze is a platform independent system which can be implemented on any robotic platform equipped with active vision systems and with any eye tracking platforms providing the required connectivity is achieved. For more information on the apparatus and the hardware architecture of TeleGaze the reader is recommended to refer to the author's previous publications on TeleGaze [Latif et al, 2008a; Latif et al, 2008b].

\section{Conclusions}

The conclusion of this work can be summarized in that the problem space of PF is not limited to one tracking algorithm or a set of robotic actions for navigation. There are a number of other tasks that need to be addressed as much as these two. Therefore, this paper presented a novel taxonomy of $\mathrm{PF}$ for mobile robots. The taxonomy shows a number of different tasks that are involved in developing any PF application. Furthermore, implementing these tasks need to be done in a natural and intuitive way in order to achieve natural HRI. The LOI of the tasks in the taxonomy might depend on the interaction scenario. Not all the tasks presented in the taxonomy might be invoked in all PF applications. However, the PF system needs to be developed so that it is capable of dealing with different tasks in the taxonomy and in different interaction scenarios. To achieve this aim, TeleGaze is integrated to a PF application. TeleGaze enables natural HRI and enables a robotic agent to understand the intentions of its human partner. The integration of TeleGaze to the PF application presented also shows an intuitive form of information acquisition for HRI applications in real life scenarios.

Also, a standardized use of both terms tracking and following is proposed and used in the taxonomy. The authors recommend the presented standardization to be used in all future publications related to PF. Finally, a novel technique for keeping a distance between the POI and the robot in PF applications is used. Through rather simple calculations and based on images from a single video camera, the initial distance between the robot and the POI is kept throughout the course of interaction using the results of the vision-based tracking algorithm.

Generalized interaction scenarios used to build the taxonomy of PF. However, TeleGaze uses one single mode of interaction. To further generalize the application domain of $\mathrm{PF}$ and to enable more natural HRI, multi-modal inter- 
action modes might be necessary. In a multimodal interaction application, each task might be invoked with different modes of interaction. Therefore, future works of the authors investigate $\mathrm{PF}$ systems that address all the tasks in the presented taxonomy using a multi-modal interaction approach.

\section{Note}

Video demonstrations of the system can be found at http://www.hemin.co.uk/

\section{References}

Benyon, D. [2005] Designing Interactive Systems: People, Activities, Contexts, Technologies, (Harlow: Adison-Wesley)

Bernardin, K., Gehrig, T., and Stiefelhagen, R. [2007] "Multi-level particle filter fusion of features and cues for audio-visual person tracking", in 2nd Annual Classification of Events Activities and Relationships (CLEAR'O') and Rich Transcription (RT'07), pp.70-81

Bhuiyan, M. A. and Liu, C. H. [2007] "Intelligent vision system for human-robot interface", in Proc. of World Academy of Science, Engineering and Technology

Bradski, G. R. [2008] Learning OpenCV: Computer vision with the Open $C V$ library, (Franham: Cambridge)

Carelli, R., Forte, G., Canali, L., Mut, V., Araguas, G. and Detefanis, E. [2008] "Autonomous and teleoperation control of a mobile robot", Mechatronics, 18, pp. 187-194

Chen, Z. and Birchfield, S. T. [2007] "Person following with a mobile robot using binocular featurebased tracking", in Proc. of IEEE/RSJ International Conference on Intelligent Robots and Systems (IROS'07), pp.815-820

Decker, D., and Piepmeier, A. J. [2008] "Gaze tracking interface for robotic control", in 40th Southeastern Symposium on System Theory, pp.274-278

Duchowski, A. T. [2002] "A breadth-first survey of eye-tracking applications", Behavior Research Methods, Instruments, and Computers, 34, pp.455-470

Goodrich, M. A. and Olsen Jr., D. R. [2003] "Seven principles of efficient human robot interaction", in Proc. of IEEE International Conference on Systems, Man and Cybernetics, pp. 3942-3948

Hu, C., Ma, X., and Dai, X. [2007] "A robust person tracking and following approach for mobile robot", in Proc. of IEEE International Conference on Mechatronics and Automation (ICMA'07), pp.3571-3576

Hyukseong, K., Youngrock, Y., Jae, B. P., and Kak, A. C. [2005] "Person tracking with a mobile robot using two uncalibrated independently moving cameras", in Proc. of IEEE International Conference on Robotics and Automation, pp.2877-83

Latif, H. O., Sherkat, N., and Lotfi, A. [2008] "TeleGaze: Teleoperation through eye gaze", in Proc. of IEEE International Conference on Cybernetics and Intelligent Systems, pp.164-169

Latif, H. O., Sherkat, N., and Lotfi, A. [2008] "Remote control of mobile robots through human eye gaze: The design and evaluation of an interface", in Proc. of SPIE Europe Security + Defence, pp.71120x

Latif, H. O., Sherkat, N., and Lotfi, A. [2009] "Fusion of automation and teleoperation for personfollowing with mobile robots", in Proc. of IEEE International Conference on Information and $\mathrm{Au}$ tomation (ICIA'09)

Mohammad, Y. and Nishida, T. [2008] "Reactive gaze control for natural human-robot interactions", in Proc. of IEEE International Conference on Robotics, Automation and Mechatronics (RAM'08), pp.47-54

Rutter, D. [1984] Looking and Seeing: The Role of Visual Communication in Social Interaction, Chicester: Wiley

Spexard, T., Li, S., Wrede, B., Fritsch, J., Sagerer, G., Booij, O., Zivkovic, Z., Terwijin, B., and Krose, B. [2006] "BIRON, where are you? enabling a robot to learn new places in a real home environment by integrating spoken dialog and visual localization", in IEEE/RSJ International Conference on Intelligent Robots and Systems, pp. 934-40

Takemura, H., Ito, K., and Mizoguchi, H. [2007] "Person following mobile robot under varying illumination based on distance and color information", in Proc. of IEEE International Conference on Robotics and Biomimetics (ROBIO'7), pp.1500-1505

Treptow, A, Cielniak, G., and Duckett, T. [2005] "Active people recognition using thermal and grey images on a mobile security robot", in IEEE/RSJ International Conference on Intelligent Robots and Systems, pp.2103-2108

Tsalatsanis, A., Valavanis, K., and Yalcin, A. [2007] "Vision based target tracking and collision avoidance for mobile robots", Journal of Intelligent and Robotic Systems: Theory and Applications, 48, pp.285-304 


\section{Author Biography}

H. O. LATIF received a BSc. degree in Civil Engineering in 1998 from University of Salahaddin and later in 2005 another BSc. degree in Computing and Statistics from University of Sulaimanee both in Kurdistan, the northern Iraq. He received a graduate diploma in Computing and Informatics from the Nottingham Trent International College (NTIC) in 2006 and following that, he commenced on studying for a $\mathrm{PhD}$ degree at the Nottingham Trent University (NTU) in England which continues to date. His scientific research activities include mobile robot teleoperation, human-robot interaction, computer and robot vision, eye tracking and intelligent user interfaces.

Prof. N Sherkat received a B.Sc Honours degree in Mechanical Engineering from University of Nottingham in 1985. He received a Ph.D. in high speed geometric processing for continuous path generation, from the Nottingham Trent
University in 1989. He is currently Associate Dean of Science and Technology at The Nottingham Trent University. His interests are intelligent pattern recognition, intelligent human computer interaction and multimodal biometrics.

Dr. A. LOTFI received his BSc and MTech. in control systems from Isfahan University of Technology, Iran and Indian Institute of Technology, India respectively. He received his $\mathrm{PhD}$ degree in Learning Fuzzy Systems from University of Queensland, Australia in 1995. He is currently a senior lecturer in School of Science and Technology, Nottingham Trent University, UK. He is the group leader for Ambient and Computational Intelligent research group. Dr LOTFI is the author of over 60 scientific papers in the area of computational intelligent and control. His main scientific research interest includes, intelligent control, computation intelligence, robotics, fuzzy logic and systems and intelligent data analysis. 